
\section{Contist}

Department of Pneumology, Katholieke Universiteit Leuven and Universitair Ziekenhuis Gasthuisberg, Leuven, Belgium

\section{Correspondence to}

Dr Jonas Yserbyt, Department of Pneumology, University

Hospital Gasthuisberg Herestraat 49, Leuven 3000,

Belgium; jonas.yserbyt@ uzleuven.be

Received 10 April 2012 Accepted 4 May 2012

To cite: Yserbyt J, Ninane V. Thorax 2013, 68, 117-118.

\title{
Aggravating cough in a patient with suspected sarcoidosis
}

\author{
Jonas Yserbyt, Vincent Ninane
}

A middle-aged patient with a recent history of a complete surgical resection of a nodular melanoma of the lower back, was referred to the clinic because of increasing exertional dyspnoea. A chest CT showed multiple enlarged mediastinal adenopathies with a micronodular interstitial pattern (figure 1). Transbronchial biopsies revealed non caseating granulomas. Moreover, because ofatrioventricular junctional arrhythmia, an MRI scan of the heart was performed, which showed myocardial granulomas. Sarcoidosis was diagnosed and treated with steroids.

Because of the aggravating cough, a second flexible bronchoscopy was performed four months later this and showed a dark brownish pigmentation lining the bronchial wall (figure 2). These lesions were predominantly located on the right side of the bronchial wall and started to bleed easily after the slightest contact.

Histopathological specimens of these endobronchial lesions showed the presence of a metastatic malignant melanoma (figure 3).

Endobronchial metastases arising from extra pulmonary solid malignant tumours are very rare, but frequently a late manifestation in the course of solid tumours. ${ }^{1}$ The latter being the reason for a poor prognosis, which reaches a few months at most. Breast, colon and renal adenocarcinomas are the tumours most frequently associated with this presentation. ${ }^{1}$ To our knowledge, endobronchial metastases, due to malignant melanoma, have not been reported in peer reviewed literature.

When localised, endobronchial treatment using a rigid bronchoscopy can alleviate symptoms such as dyspnoea, haemoptysis and retro-obstructive infections. To palliate symptoms, external radiotherapy as a stand-alone therapy or as add-on to an interventional bronchoscopy is recommended. When lesions are disseminated throughout the tracheobronchial tract, systemic chemotherapy is the only treatment modality in fit patients.

Patients with cutaneous melanomas arising in the head, neck and trunk areas have a poorer prognosis than those who have melanomas arising in the upper extremity. However, the site of the primary tumour is not as important as the factors included in the American Joint Committee on Cancer staging system (eg, tumour thickness, mitotic rate, ulceration and nodal involvement). ${ }^{2}$ Lung metastases in patients with a normal serum lactate dehydrogenase $(\mathrm{LDH})$ have a poorer prognosis than those who have skin, subcutaneous and lymphatic disease, but have a better prognosis than those with metastases to other visceral sites. One year survival rates are $63 \%, 53 \%$ and $33 \%$ respectively. If $\mathrm{LDH}$ levels are elevated at the time of the diagnosis of metastatic disease, the before mentioned survival rates show a $50 \%$ decrease. In the case presented, initial staging of the cutaneous lesion was a stage IIA (10-year survival of $65 \%){ }^{2}$

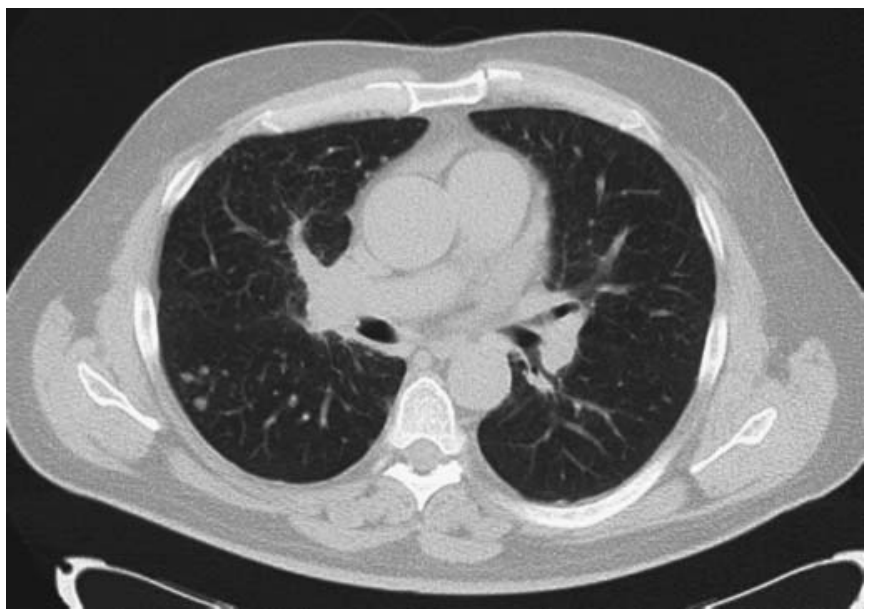

Figure 1 Chest CT scan showing an enlargement of multiple mediastinal adenopathies, with a multinodular interstitial pattern of perilymphatic distribution on top, predominantly on the right side. 


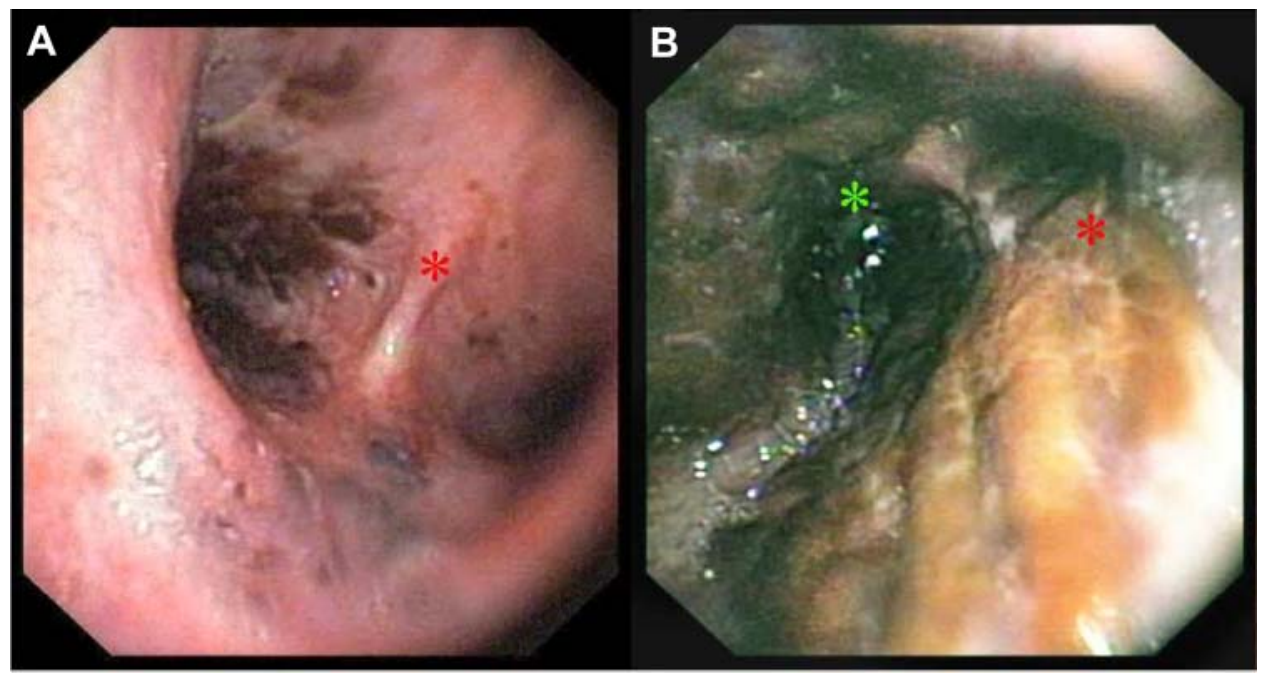

Figure 2 A) Bronchoscopic view of the main carina (asterisk) shows a dark brown pigmentation lining the bronchial wall on both sides of the main carina, predominantly located in the right main bronchus. B) Bronchoscopic view of the right main bronchus showing a subocclusion of the right upper lobe bronchus (asterisk on the left) and the orifice of the intermediate bronchus (asterisk on the right). The dark mucosal lesions are spreading from the right main bronchus down to the right lower lobe.
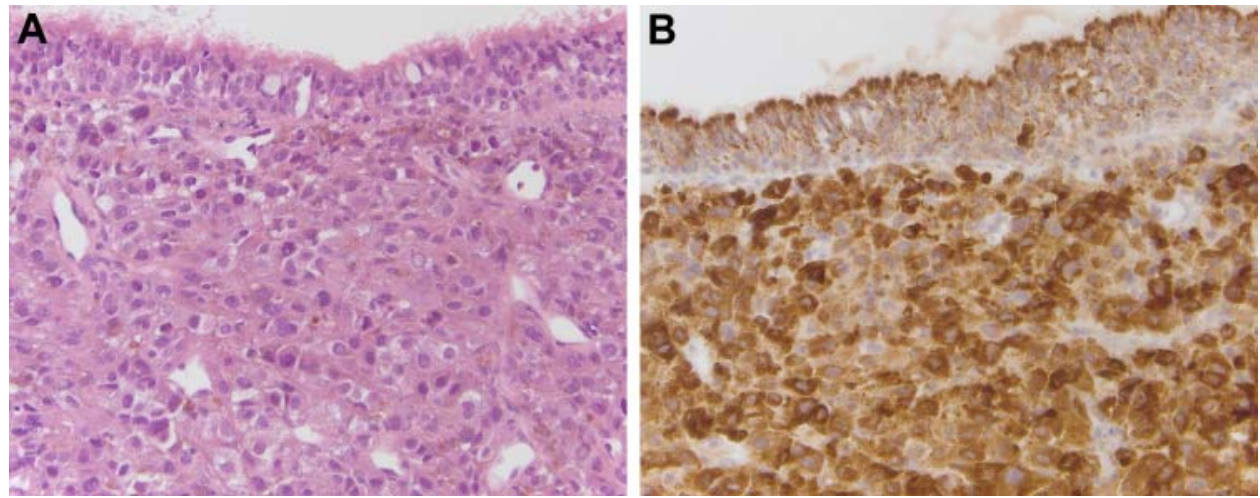

Figure 3 A) High power micrograph of bronchial tissue showing the presence of tumorous cells with large, slightly eosinophilic cytoplasm, large irregular hyperchromatic nuclei which contains large amounts of melanin. B) Staining for Hmb45 and Melan A confirmed the presence of a metastatic malignant melanoma.

At the time of detection of the endobronchial metastasis, LDH levels were elevated $(521 \mathrm{U} / \mathrm{l})$.

\section{Competing interests None.}

Patient consent Not obtained.

Provenance and peer review Not commissioned; internally peer reviewed.

\section{REFERENCES}

1 Katsimbri $\mathrm{P}$, Bamias A, Froudarakis $\mathrm{M}$, et al. Endobronchial metastases secondary to solid tumors: report of eight cases and review of the literature. Lung Cancer 2000;28:163-70

2 Balch C, Gershenwald J, Soong S, et al. Final version of 2009 AJCC melanoma staging and classification. J Clin Oncol 2009;27:6199-206. 